\title{
Ultrasound Guided Versus CT-Controlled Pararadicular Injections in the Lumbar Spine: A Prospective Randomized Clinical Trial
}

\author{
A. Loizides, H. Gruber, S. Peer, K. Galiano, R. Bale, and J. Obernauer
}

\begin{abstract}
BACKGROUND AND PURPOSE: Injection therapies play a major role in the treatment of lower back pain and are to date performed mainly under CT- or fluoroscopic guidance. We conducted this study to evaluate the accuracy, time savings, radiation doses, and pain relief of US-guided pararadicular injections versus CT-controlled interventions in the lumbar spine in a prospective randomized clinical trial.
\end{abstract}

MATERIALS AND METHODS: Forty adult patients were consecutively enrolled and assigned to a US or CT group. US-guided pararadicular injections were performed on a standard US device by using a broadband curved-array transducer (9-4 or 5-1 MHz). In the in-plane technique, the needle was advanced through the respective segmental intertransverse ligament. The needle tip position was verified by $\mathrm{CT}$. The CT-guided approaches were performed under standardized procedures by using the CT-positioning laser function.

RESULTS: The accuracy of US-guided interventions was $90 \%$. The mean time to final needle placement in the US group was $4.0 \pm 1.8$ minutes, and in the CT group, $7.6 \pm 2.1$ minutes. The mean radiation doses, including CT confirmation for study purposes only, were $20.3 \pm$ $9.0 \mathrm{mGy} \mathrm{cm}$ for the US group and $42.6 \pm 36.1 \mathrm{mGy} \mathrm{cm}$ for the CT group. Both groups showed the same significant pain relief $(P<.05)$ without relevant "intermethodic" differences of pain relief $(P>.05)$.

CONCLUSIONS: US-guided pararadicular injections show a therapeutic effect similar to that in the time-consuming, expensive, ionizing CT or fluoroscopically guided pararadicular injections and result in a significant reduction of procedure time expenditure and avoidance of radiation.

ABBREVIATIONS: PAP = pararadicular aditus plane; US = ultrasound; VAS = Visual Analog Scale

I. njection therapies play a major role in treatment and rehabilitation of patients with back pain. ${ }^{1,2}$ Low back pain and radiculopathy are very common conditions and are at least in part due to our modern lifestyle. In fact, most individuals will experience neck and/or low back pain at least once in their lives, and with increasing age, a greater number of patients with such symptoms are seen by family physicians and in outpatient clinics. ${ }^{3-6}$ Aside from physical therapy and other rehabilitative methods, injection therapies targeted to the nerve roots are well-established in the treatment of lumbar radiculopathy and have been performed without image guidance for many years. Currently, minimally invasive imaging-guided techniques have entered the toolbox of the pain physician and, because of their ease of use and much

Received March 12, 2012; accepted after revision May 9.

From the Departments of Radiology (A.L., H.G., S.P., R.B.) and Neurosurgery (K.G. J.O.), Innsbruck Medical University, Innsbruck, Austria.

Please address correspondence to Alexander Loizides, MD, Department of Radiology, Innsbruck Medical University, Anichstra 35, 6020 Innsbruck, Austria; e-mail: alexander.loizides@i-med.ac.at

http://dx.doi.org/10.3174/ajnr.A3206 better success rates compared with "blind" injections, are becoming an integral part of multidisciplinary pain management. ${ }^{1,2}$ To date, injection therapies are preferentially performed as fluoroscopically or CT-guided interventions..$^{7-11}$ CT allows a precise localization of the needle (tip) by providing precise axial anatomic data and some soft-tissue resolution. Thus, facet joint injections can be performed more accurately under CT than under fluoroscopic guidance. ${ }^{12}$ Nevertheless, both guidance modalities deliver significant radiation doses, rely on the use of equipment that is expensive and often unavailable, and render the therapist dependent on the radiologist.

US has proved to be sufficiently reliable and accurate in the demonstration of lumbar paravertebral anatomy, ${ }^{13-17}$ and the basic feasibility of US-guided injection therapy at the spine was demonstrated in several studies. ${ }^{13-16,18-24}$ US provides real-time guidance, is relatively inexpensive, does not produce ionizing radiation, and is broadly available.

The aim of this prospective randomized clinical trial was to evaluate the accuracy (technical precision), the overall effect on pain relief, patient discomfort (in respect to radiation 
dose), and time savings of US-guided pararadicular injections in the lumbar spine compared with CT-controlled interventions.

\section{MATERIALS AND METHODS Patients}

The study protocol of this prospective randomized analysis was reviewed and approved by the institutional ethics board of Innsbruck Medical University. Patients were selected consecutively in the outpatient department of the Department of Neurosurgery on the basis of a standard clinical neurologic examination and

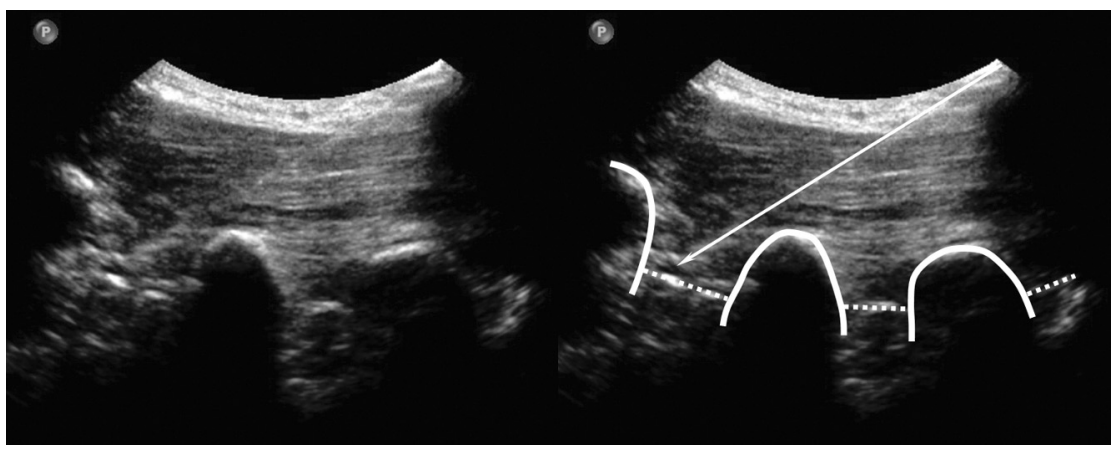
functional testing. Forty adult patients (21 women and 19 men) were consecutively enrolled.

All patients met the following inclusion criteria:

1) Had clinical-radiologic signs of low back pain without paresis

2) Were older than 19 years

3) Gave informed consent to inclusion in the study

4) Had current CT or MR imaging of their lumbar spine available.

Patients were excluded for the following reasons:

1) Had an allergy to steroids or anesthetics

2) Had a body mass index of $>35 \mathrm{~kg} / \mathrm{m}^{2}$ (Reference 19)

3) Had a spinal infection or a local or systemic infection elsewhere in the body

4) Were pregnant

5) Had diabetes

6) Had spinal tumors

7) Were under anticoagulation therapy or had uncorrectable coagulopathy.

The referring physician, who evaluated the patients, selected the level for the pararadicular injection on the basis of standard diagnostic and clinical methods. Plain radiographs, CT scans, or MR images excluded potential contraindications to an injection procedure, such as a spinal tumor, instability, diskitis, disk extrusion, fracture, and so forth. Before randomization, a VAS regarding the patient's current perception of low back pain was obtained. Using a computer-generated randomization table, we assigned patients to 1 of 2 groups. One group consisted of patients scheduled for US-guided injections, and the second group of patients was scheduled for CT-controlled injections.

\section{Ultrasound Guided Procedure}

After preparing the instruments under sterile conditions and covering the US transducer with a sterile cover, we placed patients in a prone position on the CT table and the region of interest was cleansed. Because patient preparation and cleansing is independent of the type of procedure, the end of cleansing was defined as the starting point for measurements of procedure time. One radiologist with experience in musculoskeletal US performed the US-guided lumbar approaches. Interventions were performed on a standard US device (iU22; Philips Healthcare, Bothell, Wash-

ington) by using either a broadband curved-array transducer working at 9-4 or 5-1 MHz. Sterile US gel was spread on the skin. The pararadicular compartment was then identified according to our recently published systematic procedure ${ }^{25}$ :

1) In a midline scan along the spinous processes, the typical transition from the first sacral to the fifth lumbar spinous process was identified. After identification of the fifth lumbar spinous process, the respective spinal segment for the injection was localized by cephalad counting of the spinous processes.

2) From a midline position, the transducer was offset laterally in a paravertebral parasagittal orientation toward the transition from the vertebral arch to the zygapophyseal joint.

3) The transducer was advanced further until the transverse processes were shown and back toward midline until the edge of the zygapophyseal joint was seen. In this final scanning plane (called the PAP), the intertransverse ligament was seen as a thin hyperechoic band between 2 adjacent transverse processes. The corresponding spinal nerve itself-if identified at all-was presented in the PAP under the intertransverse ligament as a faint slightly hypoechoic roundish structure surrounded by hyperechoic fat.

A spinal needle (21-ga, 80-mm; Sterican; Braun, Kronberg, Germany) was then inserted with an in-plane technique (needle advanced strictly parallel to the long axis of the transducer at an angle of approximately $45^{\circ}$, which enables real-time visualization of the entire needle path) (Fig 1). The needle was advanced until the targeted segmental intertransverse ligament was penetrated by the needle tip and thus, the orifice of the needle had reached the pararadicular compartment. To evaluate accuracy, a CT scan controlled this final needle position (Somatom Sensation Open; Siemens, Erlangen, Germany). First, a topogram centered on the needle tip was made for control of the correct spinal segment, and axial sections were obtained for control of needle tip position. If the needle diverged from the intended target position, it was replaced under US guidance. Once the needle was positioned correctly, the elapsed time for the procedure was recorded and 1-mL of betamethasone (4 mg) was injected into the pararadicular compartment.

\section{CT-Controlled Procedure}

Patients were prepared as specified above for the US procedure. According to the protocol, procedure time was recorded from 
Patient-related preinterventional data for both groups ${ }^{\mathrm{a}}$

\begin{tabular}{lcc}
\hline & CT & US \\
\hline Female/male & $10: 10$ & $11: 9$ \\
Age (yr) & $55 \pm 15$ & $59 \pm 16$ \\
Weight (kg) & $77 \pm 14$ & $74 \pm 16$ \\
Body mass index & $26.7 \pm 2.8$ & $25.7 \pm 4.7$ \\
Visual Analog Scale & $65 \pm 21$ & $69 \pm 22$ \\
\hline
\end{tabular}

${ }^{a}$ Sex, age, body mass index, Visual Analog Scale, and weight are reported as means. Differences between groups were not significant $(P>.05)$.

completion of patient preparation. A radiopaque marker was placed on the skin at the indicated level. A low-dose topogram $(120 \mathrm{kV}, 120 \mathrm{~mA})$ through the area of interest was obtained at 3-mm increments for a precise definition of the needle pathway. The proper access route (defined by the intended target position and angle of needle approach) was selected on the basis of these data. One radiologist with several years of experience in guided interventions performed the CT-controlled lumbar spine approaches. The cutaneous access point and needle pass were marked on the patient's skin by the CT-positioning laser function. A spinal needle (21 ga, $80 \mathrm{~mm}$, Sterican) was advanced toward the pararadicular compartment along the planned needle path and to the desired depth. Several CT sections were obtained to control needle tip advancement (CT fluoroscopy). If necessary, the needle was repositioned under CT control until it was positioned correctly. The time elapsed was recorded for the whole procedure, and again $1-\mathrm{mL}$ of betamethasone (4 mg) was injected in the pararadicular compartment.

\section{Measurements}

For both groups, time was reported in minutes, and radiation dose, as dose-length product. For follow-up of the effect of the intervention on back pain, a visual analog scale was repeated 1 month after the procedure. Accuracy of the needle tip position was verified by CT for sonographically and CT-guided interventions.

\section{Statistical Analysis}

Data were analyzed according to the intention-to-treat principle. The primary outcome was accuracy and time to final needle placement. The trial was designed to detect an absolute difference of 1 SD with a power of $80 \%$ at a 2 -sided significance level of .05 and a maximal drop-out rate of $20 \%$. Comparisons between groups were performed with an unpaired $t$ test for all parametric values. All $P$ values were calculated for 2 -sided tests. For the evaluation of VAS changes, we used an ANOVA with repeat measurements. For presentation, box-and-whisker plots were built whenever these were considered useful (VAS decay and time consumption).

\section{RESULTS}

After randomization, we did not observe any statistically significant difference between both groups (US versus CT group) concerning patient perception of pain and pain relief $(P=.512$ for preinterventional VAS and $P=.242$ at 6 -month control), age $(P=.36)$, body weight $(P=.53)$, and body mass index $(P=.47)$ (Table).

According to our data, the accuracy of US-guided interventions was $90 \%$ as confirmed by CT. In 2 subjects, the

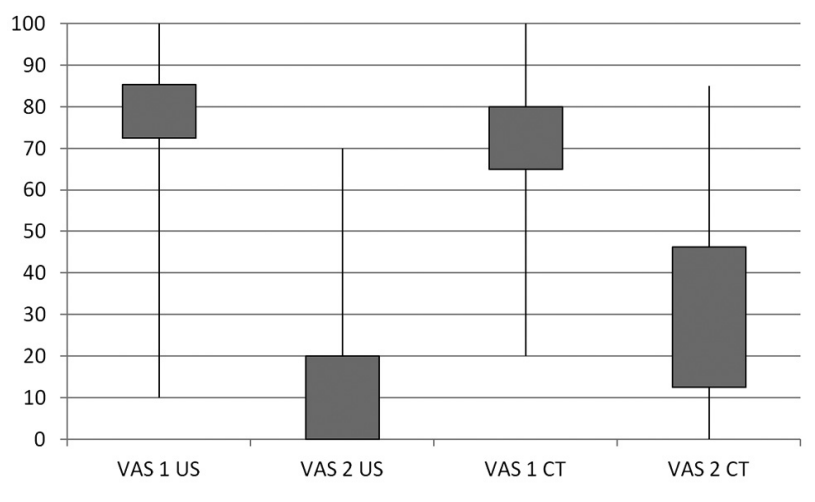

FIG 2. Boxplot of median values and SDs of pain-intensity levels indicated by the patients on the VAS. The first column of each group represents the preinterventional evaluation (VASI), and the second column of each group shows the postinterventional pain course after 1 month (VAS2). CT indicates CT-guided interventions; US, ultrasoundguided interventions.

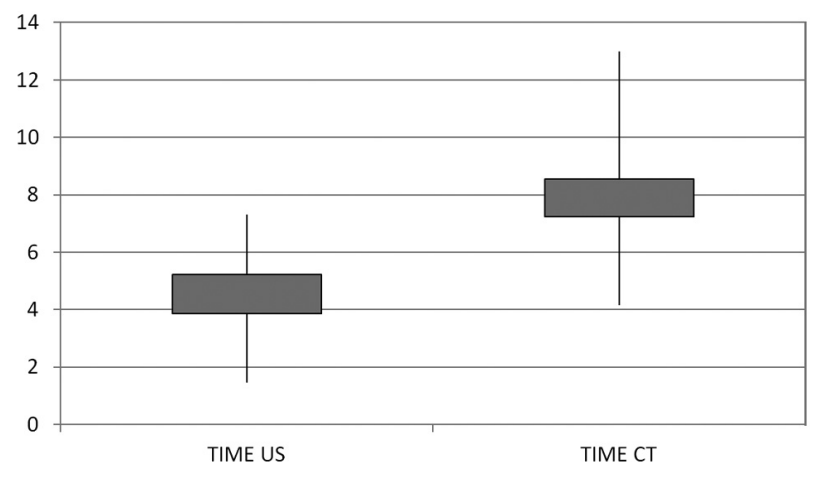

FIG 3. Boxplot of median values and SDs of procedure time. CT indicates CT-guided interventions; US, ultrasound-guided interventions.

needle had to be repositioned. The repositioning was done under US guidance, and correct replacement was then confirmed by CT. Both groups showed a significant benefit from pararadicular injections (Fig 2). Significant pain relief was found in the US-intervention group with a significant VAS decay $(P=.000000004)$ and in the CT-intervention group $(P=.000003183)$. No unintended reaction or complication was observed or noticed by any subject. The mean time to final needle placement in the US group was $4.0 \pm 1.8$ minutes; in the CT group, it was $7.6 \pm 2.1$ minute (Fig $3)$. The mean radiation doses, including CT confirmation for study purposes only, were $20.3 \pm 9.0 \mathrm{mGy} \mathrm{cm}$ for the US group and $42.6 \pm 36.1 \mathrm{mGy} \mathrm{cm}$ for the CT group.

\section{DISCUSSION}

This is the first study comparing US-guided pararadicular injections in the lumbar spine with a CT-controlled procedure in a prospective randomized clinical trial. Both patient groups showed the same significant benefit from both methods of pararadicular injection. In 90\% (18/20) of the US-guided cases, the correct needle placement was confirmed by the subsequent CT control. In 2 subjects with markedly segmental arthritic changes at the lumbar spine (which were bony appositions hampering the needle feed), the control CT diagnosed insufficiently (ie, not in the pararadicular compartment) positioned needle tips. Although the subsequent repositioning was done under US guidance alone and cor- 
rect replacement was then confirmed by CT, the deviation would not have been found if the intervention had been performed by US alone. However, in both patients who needed needle repositioning, the cumulative radiation dose, also due to 2-fold positioning control by CT, remained lower than the mean dose of patients who exclusively underwent a CT-guided intervention, though this finding should be irrelevant for daily routine. Therefore, the proposed US-guided procedure proved valid and reliable in the context of the intention-to-treat principle. If 1 of 10 subjects scheduled for a pararadicular injection had an injection failure due to this proposed US algorithm, the dose savings should still favor US over CT because at least 9 subjects should expect the same pain relief by US or CT guidance but without radiation (time savings not even taken into account).

We believe that this rather favorable result can be attributed to a set of technical factors: The sonographic technique of the inplane needle approach (where the needles are advanced strictly parallel to the long axis of the US transducer) provides real-time monitoring of the inserted needle along its entire length and path. However, sufficient training in the handling of the transducer is required to achieve constantly good visualization of the entire needle. ${ }^{26}$ A disadvantage (or even advantage) in this context might be the freehand technique needed to insert the needle correctly and to bring the needle tip to the point of injection (or variably, the most favorable path can be selected in real-time). ${ }^{27}$ The fact that US-guided pararadicular injections can be performed rapidly is not surprising. Because of the immediate visibility of the targeted intertransverse ligament, the needle can be advanced to the target structure in just a few seconds and under safe real-time controlled conditions. This finding is the greatest difference in comparison with CT-guided injections: Calculations on entry point and puncture angle of the needle have to be performed preliminarily, which require a certain amount of time.

Another disadvantage of CT-guided injections is the exposure of the patient and even the operator to radiation, if performed as CT fluoroscopy. Pararadicular injections are usually needed and performed repeatedly, some even several times per month. This requirement results in a rather high cumulative radiation dose even if the dose for 1 single CT-guided injection may be low compared with a diagnostic CT.

Still, CT is the most reliable and straightforward method for the assessment of bony details. It may theoretically be applied in any patient regardless of physical constitution and allows a precise localization of a needle by providing unrivaled axial anatomic data, but is it necessary for the proposed topic? Is it exact enough to install the therapeutic agent into the correct compartment in sufficient dose? Concerning our data, it is. No statistically significant difference counting for one or the other technique was detected; however, the $P$ value of our tests on VAS decay (highly significant for both modalities) was almost on the factor 1000 times more advantageous for the US-guided procedures, which at least could give a hint at what should happen when much larger cohorts are compared.

Fluoroscopy has a complication rate of $5 \%-10 \%$, whereas with $\mathrm{CT}$, the complication rate is approximately $0.5 \%$. Moreover, lifethreatening complications, such as pleural perforation and pneumothorax, have been described after fluoroscopically guided in- filtrations but have never been described with CT guidance; ${ }^{12}$ thus, for this feasibility study, we chose CT as the control guidance tool for our US group because-according to available literature-fluoroscopy was considered outdated.

However, our proposed technique has 1 main (but actually theoretic) disadvantage: Even under optimized scanning conditions, the lumbar roots are only seen by chance. Thus our needle path is never directed toward a lumbar root but toward the respective intertransverse ligament as an indirect landmark, which is punctured to install the therapeutic agent into the pararadicular compartment. In this context, exact adherence to the proposed PAP (ie, an injection plane closer to the neural foramen) is of utmost importance because the lumbar roots always lie next to the intertransverse ligament. Although the spinal nerve roots were not directly visualized with US guidance, the CT examination confirmed correct needle placement within the pararadicular compartment; any inadvertent puncture of a spinal nerve was excluded, in addition to imaging, on the basis of clinical observation of the patient during and after the procedure. The avoidance of puncture was assumed by the patient lacking specific segmental pain sensations during needle advancement. For the present study, the topographic basics of correct needle advancement and placement have already been defined in the study of Loizides et al. ${ }^{25}$ In that study, lumbar pararadicular injections were performed in an embalmed cadaver using the above-mentioned algorithm.

In our study, we used only a steroid for pararadicular injections. This study was not primarily designed to assess the effect of steroids in pararadicular injections. It was aimed at demonstrating a novel and safe technique for approaching the pararadicular compartment by using US.

Additionally, the equipment for image-guided interventions (CT or even fluoroscopic guidance) is rather expensive. In contrast, US devices necessary for the proposed US procedure are rather economical and broadly available. Several benefits of US as a useful adjunct during various forms of injection applications have already been shown: imaging of the individual anatomic parts, real-time needle guidance, visualization of the spread of local anesthetics, minimal risk of complications, dose reduction of local anesthetics, and shortening of onset time. ${ }^{16,20-22,28}$

\section{CONCLUSIONS}

The US approach to the pararadicular compartment in the lumbar spine is feasible and has minimal risk in the large majority of patients. It results in a significant time and radiation-dose reduction with the same benefit as CT-guided interventions and a comparable quality of instillation procedure.

\section{REFERENCES}

1. Kim PS. Role of injection therapy: review of indications for trigger point injections, regional blocks, facet joint injections, and intraarticular injections. Curr Opin Rheumatol 2002;14:52-57

2. Carrino JA, Morrison WB, Parker L, et al. Spinal injection procedures: volume, provider distribution, and reimbursement in the U.S. Medicare population from 1993 to 1999. Radiology 2002;225:723-29

3. Bogduk N. On the definitions and physiology of back pain, referred pain, and radicular pain. Pain 2009;147:17-19

AJNR Am J Neuroradiol 34:466-70 Feb 2013 www.ajnr.org 
4. Moore RA, Straube S, Derry S, et al. Chronic low back pain analgesic studies—a methodological minefield. Pain 2010;149:431-34

5. O'Neill S, Graven-Nielsen T, Manniche C, et al. Ultrasound guided, painful electrical stimulation of lumbar facet joint structures: an experimental model of acute low back pain. Pain 2009;144:76-83

6. Schiltenwolf M, Schneider S. Activity and low back pain: a dubious correlation. Pain 2009;143:1-2

7. Derby R, Kine G, Saal JA, et al. Response to steroid and duration of radicular pain as predictors of surgical outcome. Spine (Phila $\mathrm{Pa}$ 1976) 1992;17:S176-83

8. Gangi A, Dietemann JL, Mortazavi R, et al. CT-guided interventional procedures for pain management in the lumbosacral spine. Radiographics 1998;18:621-33

9. Vallee JN, Feydy A, Carlier RY, et al. Chronic cervical radiculopathy: lateral-approach periradicular corticosteroid injection. Radiology 2001;218:886-92

10. Cyteval C, Thomas E, Decoux E, et al. Cervical radiculopathy: open study on percutaneous periradicular foraminal steroid infiltration performed under CT control in $\mathbf{3 0}$ patients. AJNR Am J Neuroradiol 2004;25:441-45

11. Chang A, Pochert S, Romano C, et al. Safety of $\mathbf{1 0 0 0}$ CT-guided steroid injections with air used to localize the epidural space. AJNR Am J Neuroradiol 2011;32:E175-77

12. Aguirre DA, Bermudez S, Diaz OM. Spinal CT-guided interventional procedures for management of chronic back pain. J Vasc Interv Radiol 2005;16:689-97

13. Galiano K, Obwegeser AA, Bodner G, et al. Ultrasound guidance for facet joint injections in the lumbar spine: a computed tomographycontrolled feasibility study. Anesth Analg 2005;101:579-83, table of contents

14. Galiano K, Obwegeser AA, Bodner G, et al. Real-time sonographic imaging for periradicular injections in the lumbar spine: a sonographic anatomic study of a new technique. J Ultrasound Med 2005;24:33-38

15. Kirchmair L, Entner T, Wissel J, et al. A study of the paravertebral anatomy for ultrasound-guided posterior lumbar plexus block. Anesth Analg 2001;93:477-81, 4th contents page

16. Cotti E, Campisi G, Ambu R, et al. Ultrasound real-time imaging in the differential diagnosis of periapical lesions. Int Endod $J$ 2003;36:556-63

17. Galiano K, Obwegeser AA, Bodner G, et al. Ultrasound-guided facet joint injections in the middle to lower cervical spine: a CT-controlled sonoanatomic study. Clin J Pain 2006;22:538-43

18. Galiano K, Obwegeser AA, Bodner G, et al. Ultrasound-guided periradicular injections in the middle to lower cervical spine: an imaging study of a new approach. Reg Anesth Pain Med 2005;30:391-96

19. Greher M, Scharbert G, Kamolz LP, et al. Ultrasound-guided lumbar facet nerve block: a sonoanatomic study of a new methodologic approach. Anesthesiology 2004;100:1242-48

20. Kapral S, Krafft P, Eibenberger K, et al. Ultrasound-guided supraclavicular approach for regional anesthesia of the brachial plexus. Anesth Analg 1994;78:507-13

21. Marhofer P, Schrogendorfer K, Koinig H, et al. Ultrasonographic guidance improves sensory block and onset time of three-in-one blocks. Anesth Analg 1997;85:854-57

22. Marhofer P, Schrogendorfer K, Wallner T, et al. Ultrasonographic guidance reduces the amount of local anesthetic for 3-in-1 blocks. Reg Anesth Pain Med 1998;23:584-88

23. Kirchmair L, Entner T, Kapral S, et al. Ultrasound guidance for the psoas compartment block: an imaging study. Anesth Analg 2002;94: 706-10, table of contents

24. Galiano K, Obwegeser AA, Bale R, et al. Ultrasound-guided and CTnavigation-assisted periradicular and facet joint injections in the lumbar and cervical spine: a new teaching tool to recognize the sonoanatomic pattern. Reg Anesth Pain Med 2007;32:254-57

25. Loizides A, Gruber H, Peer S, et al. A new simplified sonographic approach for pararadicular injections in the lumbar spine: a CTcontrolled cadaver study. AJNR Am J Neuroradiol 2011;32:828-31

26. Phal PM, Brooks DM, Wolfe R. Sonographically guided biopsy of focal lesions: a comparison of freehand and probe-guided techniques using a phantom. AJR Am J Roentgenol 2005;184:1652-56

27. Bradley MJ. An in-vitro study to understand successful free-hand ultrasound guided intervention. Clin Radiol 2001;56:495-98

28. Kapral S, Krafft P, Gosch M, et al. Ultrasound imaging for stellate ganglion block: direct visualization of puncture site and local anesthetic spread - a pilot study. Reg Anesth 1995;20:323-28 